\title{
Mass spectra of singly heavy baryons in a self-consistent chiral quark-soliton model
}

\author{
June-Young Kim, ${ }^{1, *}$ Hyun-Chul Kim, ${ }^{1,2,3, \dagger}$ and Ghil-Seok Yang ${ }^{4, \$}$ \\ ${ }^{1}$ Department of Physics, Inha University, Incheon 22212, Republic of Korea \\ ${ }^{2}$ Research Center for Nuclear Physics (RCNP), Osaka University, Ibaraki, Osaka 567-0047, Japan \\ ${ }^{3}$ School of Physics, Korea Institute for Advanced Study (KIAS), Seoul 02455, Republic of Korea \\ ${ }^{4}$ Department of Physics, Soongsil University, Seoul 06978, Republic of Korea
}

(Received 30 January 2018; published 6 September 2018)

\begin{abstract}
We investigate the mass spectra of the lowest-lying singly heavy baryons, based on the self-consistent chiral quark-soliton model. We take into account the rotational $1 / N_{c}$ and strange current quark mass $\left(m_{\mathrm{s}}\right)$ corrections. Regarding $m_{\mathrm{s}}$ as a small perturbation, we expand the effective chiral action to the second order with respect to $m_{s}$. The mass spectra of heavy baryons are computed and compared with the experimental data. Fitting the classical masses of the heavy baryon to the center mass of each representation, we determine the masses of all the lowest-lying singly heavy baryons. We predict the mass of the $\Omega_{b}^{*}$ baryon to be $6081.9 \mathrm{MeV}$, when the second-order $m_{\mathrm{s}}$ corrections are included.
\end{abstract}

DOI: 10.1103/PhysRevD.98.054004

\section{INTRODUCTION}

Interest in heavy baryons is renewed as a series of new experimental data on them was reported [1-10]. A conventional heavy baryon is composed of a heavy quark and two light quarks. Since the mass of the heavy quark is very large in comparison to that of the light quarks, we can take the limit of the infinitely heavy mass of the heavy quark, i.e., $m_{Q} \rightarrow \infty$. This leads to the conservation of the heavyquark spin $\boldsymbol{J}_{Q}$, which results also in the conservation of the total spin of light quarks, $\boldsymbol{J} \equiv \boldsymbol{J}^{\prime}-\boldsymbol{J}_{Q}$, where $\boldsymbol{J}^{\prime}$ is the spin of the heavy baryon [11-13]. The conservation of the quark spins is called heavy-quark spin symmetry that makes $J$ a good quantum number. In this limit, the heavy quark can be regarded merely as a static color source, and dynamics inside a heavy baryon is mostly governed by the light quarks consisting of it. Thus, the two light quarks determine to which flavor $\mathrm{SU}(3)_{\mathrm{f}}$ representation a heavybaryon belongs. There are two different representations: $\mathbf{3} \otimes \mathbf{3}=\overline{\mathbf{3}} \oplus \mathbf{6}$. The antitriplet $(\overline{\mathbf{3}})$ has $J=0$ and total $J^{\prime}=1 / 2$, whereas the sextet $(\mathbf{6})$ has $J=1$. Thus, the spin of a heavy baryon is determined by the spin alignment of the light-quark pair together with a heavy quark. It becomes

\footnotetext{
*junyoung.kim@inha.edu

hchkim@inha.ac.kr

ghsyang@ssu.ac.kr
}

Published by the American Physical Society under the terms of the Creative Commons Attribution 4.0 International license. Further distribution of this work must maintain attribution to the author(s) and the published article's title, journal citation, and DOI. Funded by SCOAP ${ }^{3}$. either $J^{\prime}=1 / 2$ or $J^{\prime}=3 / 2$. So, there are 15 different lowest-lying heavy baryons classified as shown in Fig. 1 in the case of charmed baryons.

The masses of singly heavy baryons have been studied within various chiral solitonic models, in particular, based on bound-state approaches [14,15]. The model was originally applied to hyperons, the strange quark being regarded as a heavy one. This bound-state approach was employed to describe charmed baryons as soliton- $D$ meson bound states [16]. In the advent of heavy-quark symmetry, Refs. [17-20] incorporated this symmetry and described singly heavy baryons as a bound state of a soliton and heavy mesons. Moreover, in the original bound-state approach, the whole soliton-heavy meson bound state was quantized collectively, whereas Refs. [17-20] first quantized the soliton and then coupled it to heavy mesons. In Ref. [21], it was shown that these two different quantization schemes in the boundstate approach are in fact equivalent.

In the chiral quark-soliton model ( $\chi \mathrm{QSM})$, singly heavy baryons were examined only very recently. Reference [22] put forward a mean-field approach to describe the masses of singly heavy baryons, being motivated by Ref. [23]. The main idea of this mean-field approach is rooted in Refs. [24,25], in which Witten suggests that in the limit of the large number of colors $\left(N_{c}\right)$ the nucleon can be viewed as a bound state of $N_{c}$ valence quarks in a pion mean field with a hedgehog symmetry [26,27], as the quantum fluctuation around the saddle point of the pion field is $1 / N_{c}$ suppressed. In this large $N_{c}$ limit, the presence of $N_{c}$ valence quarks that constitute the lowest-lying baryons brings about the vacuum polarization, which produces the pion mean field. The $N_{c}$ valence quarks are also self-consistently influenced by this 


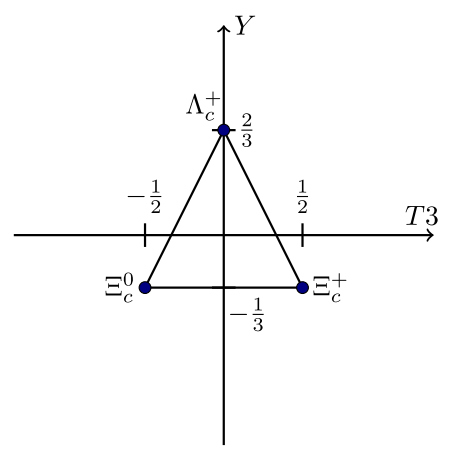

$\left(\overline{3}, J^{\prime}=\frac{1}{2}\right)$

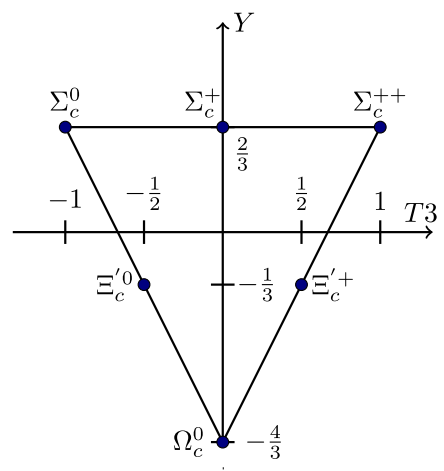

$\left(6, J^{\prime}=\frac{1}{2}\right)$

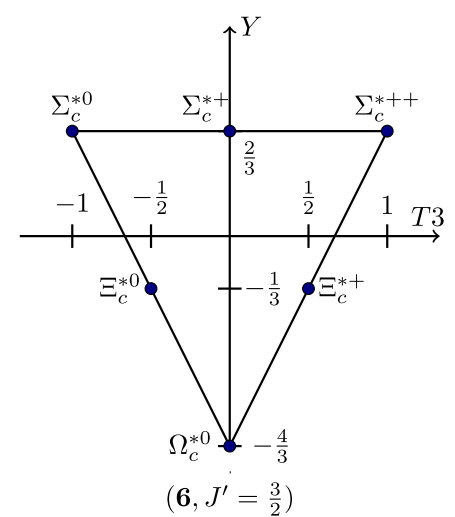

FIG. 1. The antitriplet $(\overline{\mathbf{3}})$ and sextet (6) representations of the lowest-lying heavy baryons. The left panel draws the weight diagram for the antitriplet with the total spin $1 / 2$. The centered panel corresponds to that for the sextet with the total spin $1 / 2$, and the right panel depicts that for the sextet with the total spin $3 / 2$.

pion mean field. Because of the hedgehog symmetry, an $\mathrm{SU}(2)$ soliton is embedded into the isospin subgroup of the flavor $\mathrm{SU}(3)_{\mathrm{f}}$ [25], which was also employed by various chiral soliton models [28-30]. The collective quantization of the chiral soliton yields the collective Hamiltonian with effects of flavor SU(3) symmetry breaking. This mean-field approach is called the $\chi \mathrm{QSM}$ [31-33]. One salient feature of the $\chi \mathrm{QSM}$ is that the right hypercharge is constrained to be $Y^{\prime}=N_{c} / 3$ imposed by the $N_{c}$ valence quarks. This right hypercharge selects allowed representations of light baryons such as the baryon octet (8), the decuplet (10), etc. The $\chi$ QSM describes successfully the properties of the lowestlying light baryons such as the mass splittings [34], the form factors [35-38], and parton distributions [39].

In the present work, we investigate the mass spectra of singly heavy baryons in the ground states within the framework of the $\chi \mathrm{QSM}$. Since a singly heavy baryon contains $N_{c}-1$ light valence quarks, the imposed constraint $Y^{\prime}$ should be modified as $\bar{Y}=\left(N_{c}-1\right) / 3$. This allows the lowest-lying representations: the baryon antitriplet $(\overline{\mathbf{3}})$ and the baryon sextet $(\mathbf{6})$. While in Ref. [22] all dynamical parameters were fixed by using the experimental data, we will compute them here explicitly in a selfconsistent way. This explicit calculation has a certain advantage over the previous model-independent analysis. Since we calculate the valence and sea contributions separately, we can correctly consider the pion mean field that is produced only by the $N_{c}-1$ valence quarks, whereas the vacuum polarization is kept the same as in the case of light baryons. On the other hand, the model calculation suffers from a deficiency: the classical soliton mass turns out to be rather large in the model, which is a usual problem in any chiral soliton models. It means that the predicted values of baryon masses from the model tend to be systematically overestimated. Thus, we will first concentrate on the mass splittings of the lowest-lying heavy baryons in the present work, focusing on the effects of $\mathrm{SU}(3)$ symmetry breaking.
Regarding the mass of the strange current quark as a small perturbation, we first consider its linear-order corrections to the masses of heavy baryons and then take into account the second-order corrections. However, a caveat on the second-order corrections should be mentioned. In principle, the effective chiral action may include a term that is proportional to the square of the current quark masses. However, so far, any rigorous theoretical method for that is not known. Thus, the second-order corrections in the present work imply only the contributions arising from the second-order perturbation theory. Bearing this warning in mind, we will examine the masses of both the singly charmed and bottom baryons. Taking a practical point of view, we fix the center masses in each representation by using the experimental data as in Ref. [22]. Then, we are able to produce all the values of the lowest-lying singly heavy baryons. We also predict the mass of the $\Omega_{b}^{*}$ baryon, of which the value is experimentally yet unknown.

The structure of the present work is sketched as follows. In Sec. II, we briefly review the $\chi \mathrm{QSM}$ for singly heavy baryons. In Sec. III, we examine numerically the effects of $\mathrm{SU}(3)_{\mathrm{f}}$ symmetry breaking. We then present the prediction of the heavy-baryon masses, fixing the center masses in each representation by the data. The last section is devoted to the summary and conclusions of the present work. In the Appendices, we have compiled all necessary formulas explicitly.

\section{GENERAL FORMALISM}

A heavy quark inside a heavy baryon can be regarded as a static color source in the limit of the infinite heavyquark mass $m_{Q} \rightarrow \infty$. In this case, the heavy quark is only required to make the heavy baryon a color singlet. So, it can be described as the correlation function of the $N_{c}-1$ light-quark field operators in Euclidean space, defined by 


$$
\begin{aligned}
\Pi_{B}(0, T)= & \left\langle J_{B}(0, T / 2) J_{B}^{\dagger}(0,-T / 2)\right\rangle_{0} \\
= & \frac{1}{Z} \int \mathcal{D} U \mathcal{D} \psi^{\dagger} \mathcal{D} \psi J_{B}(0, T / 2) J_{B}^{\dagger}(0,-T / 2) \\
& \times e^{\int d^{4} x \psi^{\dagger}\left(i ð+i M U^{\gamma_{5}}+i \hat{m}\right) \psi},
\end{aligned}
$$

where $J_{B}$ denotes the light-quark current consisting of $N_{c}-1$ light quarks for a heavy baryon $B$,

$$
\begin{aligned}
J_{B}(\boldsymbol{x}, t)= & \frac{1}{\left(N_{c}-1\right) !} \varepsilon^{\beta_{1} \cdots \beta_{N_{c}-1}} \Gamma_{J^{\prime} J_{3}^{\prime}, T T_{3}}^{\{f} \Psi_{\beta_{1} f_{1}}(\boldsymbol{x}, t) \cdots \\
& \times \Psi_{\beta_{N_{c}-1} f_{N_{c}-1}}(\boldsymbol{x}, t) .
\end{aligned}
$$

$\beta_{i}$ represent color indices, and $\Gamma_{J^{\prime} J_{3}^{\prime}, T T_{3}}^{\left\{f_{1} \cdots f_{N_{c}-1}\right\}}$ is a matrix with both flavor and spin indices. $J^{\prime}$ and $T$ are the spin and isospin of the heavy baryon, respectively. $J_{3}^{\prime}$ and $T_{3}$ are their third components, respectively. The notation $\langle\cdots\rangle_{0}$ in Eq. (1) stands for the vacuum expectation value. $M$ denotes the dynamical quark mass, and $U^{\gamma_{5}}$ is defined as

$$
U^{\gamma_{5}}=U \frac{1+\gamma_{5}}{2}+U^{\dagger} \frac{1-\gamma_{5}}{2}
$$

with

$$
U=\exp \left[i \frac{\pi^{a} \lambda^{a}}{f_{\pi}}\right]
$$

$\pi^{a}$ represents the pseudo-Goldstone field, and $f_{\pi}$ is the pion decay constant. $\hat{m}$ is the flavor matrix of the current quark masses, written as $\hat{m}=\operatorname{diag}\left(m_{\mathrm{u}}, m_{\mathrm{d}}, m_{\mathrm{s}}\right)$. We assume in the present work isospin symmetry, i.e., $m_{\mathrm{u}}=m_{\mathrm{d}}$. The strange current quark mass will be treated perturbatively.

Integrating over the quark fields, we obtain the correlation function as

$$
\begin{aligned}
\Pi_{B}(0, T)= & \frac{1}{Z} \Gamma_{J^{\prime} J_{3}^{\prime}, T T_{3}}^{\{f} \Gamma_{J^{\prime} J_{3}^{\prime}, T T_{3}}^{\{g\}} \\
& \times \int \mathcal{D} U \prod_{i=1}^{N_{c}-1}\left\langle 0, T / 2\left|\frac{1}{D(U)}\right| 0,-T / 2\right\rangle e^{-S_{\text {eff }}(U)},
\end{aligned}
$$

where $D(U)$ is defined as

$$
D(U)=i \gamma_{4} \partial_{4}+i \gamma_{k} \partial_{k}+i M U^{\gamma_{5}}+i \hat{m}
$$

and $S_{\text {eff }}$ represents the effective chiral action written as

$$
S_{\text {eff }}=-N_{c} \operatorname{Tr} \log D(U) .
$$

The correlation function at large separation of the Euclidean time $\tau$ picks up the ground-state energies [31,32]

$$
\lim _{\tau \rightarrow \infty} \Pi_{B}(\tau) \sim \exp \left[-\left(N_{c}-1\right) E_{\mathrm{val}}+E_{\mathrm{sea}} \tau\right],
$$

where $E_{\text {val }}$ and $E_{\text {sea }}$ the valence- and sea-quark energies. The soliton mass is then derived by minimizing selfconsistently the energies around the saddle point of the chiral field $U$,

$$
\left.\frac{\delta}{\delta U}\left[\left(N_{c}-1\right) E_{\mathrm{val}}+E_{\mathrm{sea}}\right]\right|_{U_{c}}=0,
$$

which yields the soliton mass

$$
M_{\mathrm{sol}}=\left(N_{c}-1\right) E_{\mathrm{val}}\left(U_{c}\right)+E_{\mathrm{sea}}\left(U_{c}\right) .
$$

Since a singly heavy baryon contains the heavy quark, its classical mass of a heavy baryon should be expressed as

$$
M_{\mathrm{cl}}=M_{\mathrm{sol}}+m_{Q},
$$

where $m_{Q}$ is the effective heavy-quark mass that is different from that discussed in QCD and will be absorbed in the center mass of each representation, which will be discussed later.

As in the light-baryon sector, we expect that the lowestlying heavy baryons will arise from rotational excitations of the light-quark soliton, whereas the heavy quark is kept static. Keeping in mind that the $\mathrm{SU}(2)$ soliton $U_{c}(\boldsymbol{r})$ has hedgehog symmetry, we embed it into SU(3) [25]:

$$
U(\boldsymbol{r})=\left(\begin{array}{cc}
U_{c}(\boldsymbol{r}) & 0 \\
0 & 1
\end{array}\right) .
$$

To find the $1 / N_{c}$ quantum fluctuations, we need to integrate the meson fields over small oscillations of the $U(\boldsymbol{r})$ field around the saddle point. However, we will not carry out this procedure, and this is often called the meanfield approximation. On the other hand, we have to consider explicitly the rotational zero modes that are not small and cannot be neglected. Thus, we restrict ourselves to taking into account these zero modes only. Considering a slowly rotating hedgehog field $U(\boldsymbol{r})$ in Eq. (12),

$$
U(\boldsymbol{r}, t)=A(t) U(\boldsymbol{r}) A^{\dagger}(t),
$$

where $A(t)$ is an element of the flavor SU(3) matrix, we can find the collective Hamiltonian to describe heavy baryons. For a detailed formalism of the semiclassical quantization, we refer to Ref. [32]. Regarding the angular velocity of the soliton and the current strange-quark mass as small parameters, we can expand the quark propagator in Eq. (5) with respect to them. 
Having quantized the chiral soliton, we arrive at the collective Hamiltonian for singly heavy baryons,

$$
H=H_{\mathrm{sym}}+H_{\mathrm{sb}}^{(1)}+H_{\mathrm{sb}}^{(2)}
$$

where $H_{\text {sym }}$ represents the flavor SU(3) symmetric part and $H_{\mathrm{sb}}^{(1)}$ and $H_{\mathrm{sb}}^{(2)}$ are the $\mathrm{SU}(3)$ symmetry-breaking parts to the first and second orders, respectively, which will be discussed later. $H_{\text {sym }}$ is expressed as

$$
H_{\mathrm{sym}}=M_{\mathrm{cl}}+\frac{1}{2 I_{1}} \sum_{i=1}^{3} \hat{J}_{i}^{2}+\frac{1}{2 I_{2}} \sum_{a=4}^{7} \hat{J}_{a}^{2},
$$

where $I_{1}$ and $I_{2}$ denote the moments of inertia of the soliton. The explicit expressions for $I_{1,2}$ are given in Eq. (A6). The operators $\hat{J}_{i}$ are the $\mathrm{SU}(3)$ generators. In the $(p, q)$ representation of the $\mathrm{SU}(3)$ group, we find the eigenvalue of the Casimir operator $\sum_{i=1}^{8} J_{i}^{2}$ as

$$
C_{2}(p, q)=\frac{1}{3}\left[p^{2}+q^{2}+p q+3(p+q)\right] .
$$

Thus, the eigenvalues of $H_{\text {sym }}$ are obtained as

$$
\begin{aligned}
E_{\mathrm{sym}}(p, q)= & M_{\mathrm{cl}}+\frac{1}{2 I_{1}} J(J+1) \\
& +\frac{1}{2 I_{2}}\left[C_{2}(p, q)-J(J+1)\right]-\frac{3}{8 I_{2}} \bar{Y}^{2} .
\end{aligned}
$$

The right hypercharge $\bar{Y}$ is constrained to be $\left(N_{c}-1\right) / 3$, which is imposed by the $N_{c}-1$ valence quarks inside a singly heavy baryon. The wave functions of the singly heavy baryon are derived as

$$
\begin{aligned}
\psi_{B}^{(\mathcal{R})}\left(J^{\prime} J_{3}^{\prime}, J ; A\right)= & \sum_{m_{3}= \pm 1 / 2} C_{J_{Q} m_{3} J J_{3}}^{J^{\prime} J_{m_{3}}^{\prime}} \sqrt{\operatorname{dim}(p, q)} \\
& \times(-1)^{-\frac{\bar{Y}}{2}+J_{3}} D_{\left(Y, T, T_{3}\right)\left(\bar{Y}, J,-J_{3}\right)}^{(\mathcal{R}) *}(A),
\end{aligned}
$$

where

$$
\operatorname{dim}(p, q)=(p+1)(q+1)\left(1+\frac{p+q}{2}\right)
$$

Note that a similar expression can be found in Ref. [20], though its formalism is rather different from the present one. $J$ and $J_{Q}$ stand for the soliton spin and heavy-quark spin, respectively. $J_{3}$ and $m_{3}$ represent the corresponding third components, respectively. Since the spin operator for the heavy baryon is given by the addition of the soliton and heavy-quark spin operators

$$
\boldsymbol{J}^{\prime}=\boldsymbol{J}_{Q}+\boldsymbol{J}
$$

the relevant Clebsch-Gordan coefficients appear in Eq. (18). The SU(3) Wigner $D$ function in Eq. (18) is just the wave function for the quantized soliton consisting of the $N_{c}-1$ valence quarks, whereas $\chi_{m_{3}}$ is the Pauli spinor for the heavy quark. $\mathcal{R}$ stands for a $\mathrm{SU}(3)$ irreducible representation corresponding to $(p, q)$.

Since a singly heavy baryon consists of $N_{c}-1$ valence quarks, we have two irreducible representations when $N_{c}=3: \mathbf{3} \otimes \mathbf{3}=\overline{\mathbf{3}} \oplus \mathbf{6}$. Thus, we have the following representations for the lowest-lying singly heavy baryons:

$$
\begin{aligned}
& {\left[\overline{\mathbf{3}}_{0}\right]=D(0,1): \text { the anti-triplet with } J=0,} \\
& {\left[\mathbf{6}_{1}\right]=D(2,0): \text { the sextet with } J=1 .}
\end{aligned}
$$

The soliton being coupled to the heavy quark, we finally get three different representations, which have been illustrated already in Fig. 1. Since the soliton in the sextet $(J=1)$ is coupled to the heavy quark $\left(J_{Q}=1 / 2\right)$, we have two sextet representations with spins $1 / 2$ and $3 / 2$, respectively, which are degenerate. The hyperfine spin-spin interaction will lift this degeneracy.

Since a singly heavy baryon consists of $N_{c}-1$ valence quarks, the pion mean fields should be changed. In Refs. $[22,40]$, a scale factor was introduced to explain the modification of the mean field, of which the value was taken to be in the range of $1-0.66$. Because all dynamical variables being proportional to the color factor were fixed by the experimental data in Refs. [22,40], it was impossible to decompose the valence and sea parts. On the other hand, we can treat separately the valence- and sea-quark contributions in the present work. So, we will replace the $N_{c}$ factor with $N_{c}-1$ only in front of the valence part of the dynamical parameters, while we keep the sea part intact.

To describe the mass splittings of SU(3) baryons in a specific representation, we have to consider the effects of flavor SU(3) symmetry breaking, dealing with the mass of the strange current quark, $m_{\mathrm{s}}$, as a small perturbation. First, we consider the first-order corrections that are proportional to the linear $m_{\mathrm{s}}$, and then we proceed to take into account the second-order corrections. In this case, the baryon wave functions are no longer in pure states but are mixed with those of higher representations. Thus, there are two different contributions: one from the collective Hamiltonian and the other from the baryon wave functions. Both corrections will be considered second-order contributions.

\section{A. Mass splittings to the linear order}

The symmetry-breaking part of the collective Hamiltonian is given as $[32,34]$ 


$$
H_{\mathrm{sb}}^{(1)}=\frac{\bar{\Sigma}_{\pi N}}{m_{0}} \frac{m_{\mathrm{s}}}{3}+\alpha D_{88}^{(8)}+\beta \hat{Y}+\frac{\gamma}{\sqrt{3}} \sum_{i=1}^{3} D_{8 i}^{(8)} \hat{J}_{i},
$$

where

$$
\begin{gathered}
\alpha=\left(-\frac{\bar{\Sigma}_{\pi N}}{3 m_{0}}+\frac{K_{2}}{I_{2}} \bar{Y}\right) m_{\mathrm{s}}, \quad \beta=-\frac{K_{2}}{I_{2}} m_{\mathrm{s}}, \\
\gamma=2\left(\frac{K_{1}}{I_{1}}-\frac{K_{2}}{I_{2}}\right) m_{\mathrm{s}} .
\end{gathered}
$$

The first term in Eq. (22) can be absorbed into the symmetric part of the Hamiltonian, since it does not contribute to the mass splittings of heavy baryons in a given representation. The $m_{0}$ represents the averaged mass of the up and down quarks. The three parameters $\alpha, \beta$, and $\gamma$ are expressed in terms of the moments of inertia $I_{1,2}$ and $K_{1,2}$, of which the valence parts are different from those in the light-baryon sector by the color factor $N_{c}-1$ in place of $N_{c}$. The valence part of $\bar{\Sigma}_{\pi N}$ is different from the $\pi N$ sigma term by the prefactor $N_{c}-1$, that is, $\bar{\Sigma}_{\pi N}=$ $\left(N_{c}-1\right) N_{c}^{-1} \Sigma_{\pi N}$, where $\Sigma_{\pi N}=\left(m_{u}+m_{d}\right)\langle N|\bar{u} u+\bar{d} d| N\rangle=$ $\left(m_{u}+m_{d}\right) \sigma$. The explicit expressions for the moments of inertia and the $\pi N$ sigma term can be found in Appendix A. Note that their sea parts are the same as in the light-baryon sector.

Taking into account the $m_{\mathrm{s}}$ corrections to the first order, we can write the masses of the singly heavy baryons in representation $\mathcal{R}$ as

$$
M_{B, \mathcal{R}}^{Q}=M_{\mathcal{R}}^{Q}+M_{B, \mathcal{R}}^{(1)}
$$

where

$$
M_{\mathcal{R}}^{Q}=m_{Q}+E_{\mathrm{sym}}(p, q)
$$

$M_{\mathcal{R}}^{Q}$ is called the center mass of a heavy baryon in representation $\mathcal{R}$. $E_{\text {sym }}(p, q)$ is defined in Eq. (17). Note that the lower index $B$ denotes a certain baryon belonging to a specific representation $\mathcal{R}$. The upper index $Q$ stands for either the charm sector $(Q=c)$ or the bottom sector $(Q=b)$. The center masses for the antitriplet and sextet representations can be explicitly written as

$$
M_{\overline{\mathbf{3}}}^{Q}=M_{\mathrm{cl}}+\frac{1}{2 I_{2}}, \quad M_{\mathbf{6}}^{Q}=M_{\overline{\mathbf{3}}}^{Q}+\frac{1}{I_{1}},
$$

where $M_{\mathrm{cl}}$ was defined in Eq. (11). The second term in Eq. (24) denotes the linear-order $m_{\mathrm{s}}$ corrections to the heavy-baryon mass

$$
M_{B, \mathcal{R}}^{(1)}=\left\langle B, \mathcal{R}\left|H_{\mathrm{sb}}^{(1)}\right| B, \mathcal{R}\right\rangle=Y \delta_{\mathcal{R}}
$$

where

$$
\delta_{\overline{\mathbf{3}}}=\frac{3}{8} \alpha+\beta, \quad \delta_{\mathbf{6}}=\frac{3}{20} \alpha+\beta-\frac{3}{10} \gamma .
$$

The values of the matrix elements for the relevant SU(3) Wigner $D$ functions are tabulated in Appendix B. Thus, we obtain the masses of the lowest-lying singly heavy baryons,

$$
M_{B, \overline{\mathbf{3}}}^{Q}=M_{\overline{\mathbf{3}}}^{Q}+Y \delta_{\overline{\mathbf{3}}}, \quad M_{\overline{B, \mathbf{6}}}^{Q}=M_{\mathbf{6}}^{Q}+Y \delta_{\mathbf{6}},
$$

with the linear-order $m_{\mathrm{s}}$ corrections taken into account.

\section{B. Mass splittings to the second order}

We now consider the second-order $m_{\mathrm{s}}$ corrections. When we include the second-order corrections, the collective wave function of baryons is no longer in a pure state but is mixed with those in higher representations. Using the standard method of perturbation theory, we can derive the second-order $m_{\mathrm{s}}$ corrections to the baryon mass, which arise from the baryon wave functions [41],

$$
M_{B}^{(2)(\mathrm{wf})}=\sum_{\mathcal{R} \neq \mathcal{R}^{\prime}} \frac{\left|\left\langle\mathcal{R}^{\prime}, B\left|H_{\mathrm{sb}}^{(1)}\right| \mathcal{R}, B\right\rangle\right|^{2}}{M_{\mathcal{R}}^{Q}-M_{\mathcal{R}^{\prime}}^{Q}},
$$

where $\mathcal{R}^{\prime}$ denote higher representations that are different from $\mathcal{R}$. These representations are determined by the irreducible decomposition of the following products: $\overline{\mathbf{3}} \otimes \mathbf{8}=\overline{\mathbf{3}} \oplus \mathbf{6} \oplus \overline{\mathbf{1 5}} \quad$ and $\quad \mathbf{6} \otimes \mathbf{8}=\overline{\mathbf{3}} \oplus \mathbf{6} \oplus \overline{\mathbf{1 5}} \oplus \overline{\mathbf{2 4}}$. The corresponding baryon wave function is then expressed as a mixed state with those in higher representations,

$$
\left|B^{(\mathcal{R})}\right\rangle=|\mathcal{R}, B\rangle-\sum_{\mathcal{R} \neq \mathcal{R}^{\prime}} \frac{\left|\left\langle\mathcal{R}^{\prime}, B\left|H_{\mathrm{sb}}^{(1)}\right| \mathcal{R}, B\right\rangle\right|}{M_{\mathcal{R}}^{Q}-M_{\mathcal{R}^{\prime}}^{Q}}\left|\mathcal{R}^{\prime}, B\right\rangle .
$$

Explicit calculation yields the collective wave functions of the baryon antitriplet and sextet, respectively, as

$$
\begin{aligned}
& \left|B_{\overline{\mathbf{3}}_{0}}\right\rangle=\left|\overline{\mathbf{3}}_{0}, B\right\rangle+p_{15}^{B}\left|\overline{\mathbf{1 5}}_{0}, B\right\rangle, \\
& \left|B_{\mathbf{6}_{1}}\right\rangle=\left|\mathbf{6}_{1}, B\right\rangle+q_{15}^{B}\left|\overline{\mathbf{1 5}}_{1}, B\right\rangle+q_{24}^{B}\left|\overline{\mathbf{2 4}}_{1}, B\right\rangle,
\end{aligned}
$$

with the mixing coefficients

$$
p \frac{B}{15}=p_{\overline{15}}\left[\begin{array}{c}
2 \\
\sqrt{3}
\end{array}\right], \quad q_{15}^{B}=q_{\overline{15}}\left[\begin{array}{c}
2 \sqrt{2} \\
\sqrt{3} \\
0
\end{array}\right], \quad q_{24}^{B}=q_{\overline{24}}\left[\begin{array}{c}
1 \\
\sqrt{3 / 2} \\
\sqrt{3 / 2}
\end{array}\right]
$$


where

$$
\begin{aligned}
& p_{\overline{15}}=-\frac{3}{16 \sqrt{5}} \alpha I_{2}, \quad q_{\overline{15}}=-\frac{1}{4 \sqrt{5}}\left(\alpha+\frac{2}{3} \gamma\right) I_{2}, \\
& q_{\overline{24}}=-\frac{2}{25}\left(\alpha-\frac{1}{3} \gamma\right) I_{2},
\end{aligned}
$$

in the bases of $\left[\Lambda_{Q}, \Xi_{Q}\right]$ and $\left[\Sigma_{Q}, \Xi_{Q}^{\prime}, \Omega_{Q}\right]$, respectively. Then, we obtain the second-order corrections to the masses of the singly heavy baryons from the baryon wave functions as

$$
\begin{aligned}
& M_{\Lambda_{Q}}^{(2)(\mathrm{wf})}=-I_{2} \frac{9}{160} \alpha^{2}, \\
& M_{\Xi_{Q}}^{(2)(\mathrm{wf})}=-I_{2} \frac{27}{640} \alpha^{2}, \\
& M_{\Sigma_{Q}(\mathrm{wf})}^{(2)}=-I_{2} \frac{1}{90}(3 \alpha+2 \gamma)^{2}-I_{2} \frac{2}{1125}(3 \alpha-\gamma)^{2}, \\
& M_{\Xi_{Q}^{\prime}(\mathrm{wf})}^{(2)}=-I_{2} \frac{1}{240}(3 \alpha+2 \gamma)^{2}-I_{2} \frac{1}{375}(3 \alpha-\gamma)^{2}, \\
& M_{\Omega_{Q}}^{(2)(\mathrm{wf})}=-I_{2} \frac{1}{375}(3 \alpha-\gamma)^{2} .
\end{aligned}
$$

There are yet other second-order $m_{\mathrm{s}}$ corrections that come from the collective Hamiltonian [32,41],

$$
\begin{aligned}
H_{\mathrm{sb}}^{(2)}= & m_{\mathrm{s}}^{2}\left[\frac{2}{3} \frac{K_{1}^{2}}{I_{1}} \sum_{i=1}^{3} D_{8 i}^{(8)}(A) D_{8 i}^{(8)}(A)+\frac{2}{3} \frac{K_{2}^{2}}{I_{2}} \sum_{a=4}^{7} D_{8 a}^{(8)}(A) D_{8 a}^{(8)}(A)-\frac{2}{3} N_{1} \sum_{i=1}^{3} D_{8 i}^{(8)}(A) D_{8 i}^{(8)}(A)\right. \\
& \left.-\frac{2}{3} N_{2} \sum_{a=4}^{7} D_{8 a}^{(8)}(A) D_{8 a}^{(8)}(A)-\frac{2}{9} N_{0}\left(1-D_{88}^{(8)}(A)\right)^{2}\right]
\end{aligned}
$$

where $N_{0}, N_{1}$, and $N_{2}$ are defined in Appendix A. Computing the matrix elements of Eq. (36), we obtain the secondorder $m_{\mathrm{s}}$ corrections to the masses of the singly heavy baryons, which arise from the collective Hamiltonian,

$$
\begin{aligned}
& M_{\Lambda_{Q}}^{(2)(\mathrm{op})}=m_{\mathrm{s}}^{2}\left(\frac{3}{20} \frac{K_{1}^{2}}{I_{1}}+\frac{2}{5} \frac{K_{2}^{2}}{I_{2}}+\frac{13}{180} N_{0}-\frac{3}{20} N_{1}-\frac{2}{5} N_{2}\right), \\
& M_{\Xi_{Q}}^{(2)(\mathrm{op})}=m_{\mathrm{s}}^{2}\left(\frac{3}{10} \frac{K_{1}^{2}}{I_{1}}+\frac{3}{10} \frac{K_{2}^{2}}{I_{2}}-\frac{7}{90} N_{0}-\frac{3}{10} N_{1}-\frac{3}{10} N_{2}\right), \\
& M_{\Sigma_{Q}}^{(2)(\mathrm{op})}=m_{\mathrm{s}}^{2}\left(\frac{19}{90} \frac{K_{1}^{2}}{I_{1}}+\frac{16}{45} \frac{K_{2}^{2}}{I_{2}}+\frac{1}{90} N_{0}-\frac{19}{90} N_{1}-\frac{16}{45} N_{2}\right), \\
& M_{\Xi_{Q}^{\prime}}^{(2)(\mathrm{op})}=m_{\mathrm{s}}^{2}\left(\frac{4}{15} \frac{K_{1}^{2}}{I_{1}}+\frac{1}{3} \frac{K_{2}^{2}}{I_{2}}-\frac{2}{45} N_{0}-\frac{4}{15} N_{1}-\frac{1}{3} N_{2}\right), \\
& M_{\Omega_{Q}}^{(2)(\mathrm{op})}=m_{\mathrm{s}}^{2}\left(\frac{1}{3} \frac{K_{1}^{2}}{I_{1}}+\frac{4}{15} \frac{K_{2}^{2}}{I_{2}}-\frac{1}{9} N_{0}-\frac{1}{3} N_{1}-\frac{4}{15} N_{2}\right) .
\end{aligned}
$$

We will call them the second-order $m_{\mathrm{s}}$ corrections from the operator so that we distinguish them from those coming from the wave function corrections. Considering these secondorder $m_{\mathrm{s}}$ corrections, we can extend Eq. (24) to

$$
M_{B, \mathcal{R}}^{Q}=M_{\mathcal{R}}^{Q}+M_{B, \mathcal{R}}^{(1)}+M_{B, \mathcal{R}}^{(2)},
$$

where $M_{B, \mathcal{R}}^{(2)}$ denote the second-order corrections to a baryon in representation $\mathcal{R}$.

\section{RESULTS AND DISCUSSION}

We are now in a position to compute the mass splittings of the lowest-lying singly heavy baryons. Reference [34] shows in detail how model parameters such as the cutoff masses and the current quark masses can be fixed in the vacuum sector. In the present work, we choose the constituent quark mass $M=420 \mathrm{MeV}$, which provided the best prediction of baryon observables [32]. The mass of the strange current quark, $m_{s}$, was taken to be $180 \mathrm{MeV}$ also in previous works, since it describes the mass splittings of the baryon octet and decuplet. In fact, the value of the $m_{s}$ can be fixed by fitting the mass splittings of the singly heavy baryon antitriplet and sextet. The smaller values of $m_{s}$ yield better results of the mass splittings of the singly heavy baryons in comparison with those of the baryon octet and decuplet. In the bottom baryon sector, even the smaller value of $m_{s}$ is favored. Though it is interesting to see why the dropping of the $m_{s}$ value in the heavy-baryon sector is different from that in the light-quark sector, we will use the canonical value of $m_{s}=180 \mathrm{MeV}$ as in the previous works [32,35-37].

We follow Refs. [32,34] for the numerical methods of diagonalizing the Dirac equation in the presence of the pion field and deriving the self-consistent solutions of the equations of motion. However, we use a much larger size of the box in solving the one-body Dirac equation such that we are able to reduce a numerical instability and uncertainties. ${ }^{1}$ Detailed numerical techniques and relevant references are also given in Refs. [32,34].

In Table I, we list the numerical results of the moments of inertia, the $\pi N$ sigma term, and the classical soliton mass $M_{\text {sol }}$. As discussed in Sec. II, the expressions for the valence parts of all relevant quantities should be modified.

\footnotetext{
${ }^{1} 10 \mathrm{fm}$ is taken for the box size in the present work, whereas $5 \mathrm{fm}$ was used in Ref. [34].
} 
TABLE I. Numerical results of the moments of inertia, the $\Sigma_{\pi N}$ term, and the classical mass of the soliton. Note that the valence parts of the moments of inertia for singly heavy baryons have the $N_{c}-1$ factor, whereas $N_{c}$ for light baryons.

\begin{tabular}{lclr}
\hline \hline Light baryon & \multicolumn{2}{c}{ Singly heavy baryon } \\
\hline$I_{1}(\mathrm{fm})$ & 1.108 & $I_{1}(\mathrm{fm})$ & 0.844 \\
$I_{2}(\mathrm{fm})$ & 0.529 & $I_{2}(\mathrm{fm})$ & 0.404 \\
$K_{1}(\mathrm{fm})$ & 0.428 & $K_{1}(\mathrm{fm})$ & 0.286 \\
$K_{2}(\mathrm{fm})$ & 0.272 & $K_{2}(\mathrm{fm})$ & 0.181 \\
$N_{0}(\mathrm{fm})$ & 0.457 & $N_{0}(\mathrm{fm})$ & 0.499 \\
$N_{1}(\mathrm{fm})$ & 0.410 & $N_{1}(\mathrm{fm})$ & 0.380 \\
$N_{2}(\mathrm{fm})$ & 0.323 & $N_{2}(\mathrm{fm})$ & 0.286 \\
$\Sigma_{\pi N}(\mathrm{MeV})$ & 43.7 & $\bar{\Sigma}_{\pi N}(\mathrm{MeV})$ & 40.0 \\
$M_{\text {sol }}(\mathrm{MeV})$ & 1291.8 & $M_{\text {sol }}(\mathrm{MeV})$ & 1093.3 \\
\hline \hline
\end{tabular}

The prefactor $N_{c}$ in those expressions for light baryons, which counts the number of valence quarks, should be replaced by the factor $N_{c}-1$, since a singly heavy baryon consists of $N_{c}-1$ light valence quarks. So, the difference between the left panel of Table I and the right one arises from the different prefactor of each valence part. The definition of $\bar{\Sigma}_{\pi N}$ is just the same as $\Sigma_{\pi N}$ except for the valence contribution as shown in Eq. (A1).

We are not able to determine the masses of singly heavy baryons, because the center mass given in Eq. (26) in each representation seems overestimated, compared with the experimental data. In addition, we must know the hyperfine interaction that will lift the degeneracy of different spin states in the sextet representation. Thus, we will fix each center mass and parameters for the hyperfine splitting, using the experimental data such that we can determine the masses of the lowest-lying singly heavy baryons. We will follow the method proposed by Ref. [22] in which the spinspin interaction Hamiltonian is given as

$$
H_{\text {solQ }}=\frac{2}{3} \frac{\kappa}{m_{Q} M_{\mathrm{sol}}} \boldsymbol{J} \cdot \boldsymbol{J}_{Q}=\frac{2}{3} \frac{\varkappa}{m_{Q}} \boldsymbol{J} \cdot \boldsymbol{J}_{Q}
$$

where $\kappa$ represents the flavor-independent hyperfine coupling constant. Note that the baryon antitriplet does not acquire any contribution from the hyperfine interaction, since the corresponding soliton has spin $J=0$. On the other hand, the baryon sextet has $J=1$. Being coupled to the heavy-quark spin, it produces two different multiplets, i.e., $J^{\prime}=1 / 2$ and $J^{\prime}=3 / 2$, of which the masses are expressed, respectively, as

$$
M_{B, \mathbf{6}_{1 / 2}}^{Q}=M_{B, 6}^{Q}-\frac{2}{3} \frac{\varkappa}{m_{Q}}, \quad M_{B, \mathbf{6}_{3 / 2}}^{Q}=M_{B, 6}^{Q}+\frac{1}{3} \frac{\varkappa}{m_{Q}} .
$$

Thus, we find the hyperfine mass splitting,

$$
M_{B, \mathbf{6}_{3 / 2}}^{Q}-M_{B, \mathbf{6}_{1 / 2}}^{Q}=\frac{\varkappa}{m_{Q}}
$$

where the corresponding numerical value can be determined by using the center value of the sextet masses. In the charmed and bottom baryon sectors, we obtain the corresponding numerical values, respectively,

$$
\frac{\varkappa}{m_{c}}=68.1 \mathrm{MeV}, \quad \frac{\varkappa}{m_{b}}=20.3 \mathrm{MeV} .
$$

Combining Eq. (40) with Eq. (38), we can derive the final masses of the lowest-lying singly heavy baryons:

$$
\begin{aligned}
M_{B, \overline{\mathbf{3}}}^{Q} & =M_{\overline{\mathbf{3}}}^{Q}+M_{B, \overline{\mathbf{3}}}^{(1)}+M_{B, \overline{3}}^{(2)}, \\
M_{B, \mathbf{6}_{1 / 2}}^{Q} & =M_{\mathbf{6}}^{Q}+M_{B, \mathbf{6}}^{(1)}+M_{B, \mathbf{3}}^{(2)}-\frac{2}{3} \frac{\varkappa}{m_{Q}}, \\
M_{B, \mathbf{6}_{3 / 2}}^{Q} & =M_{\mathbf{6}}^{Q}+M_{B, \mathbf{6}}^{(1)}+M_{B, \mathbf{6}}^{(2)}+\frac{1}{3} \frac{\varkappa}{m_{Q}} .
\end{aligned}
$$

The numerical results of the charmed baryons are listed in Table II. As expected, the inclusion of the second-order $m_{s}$ corrections produces the results in better agreement with the experimental data. It is of interest to compare the present results with those of Ref. [22], in which the modelindependent approach was employed. Theoretically, the present approach has a certain advantage over Ref. [22], since we can consistently treat both the valence-quark and sea-quark contributions with the correct $N_{c}-1$ factor taken into account. In the model-independent analysis, an additional scale factor had to be introduced, since it was not possible to decompose each contribution into the valence and sea parts $[40,42,43]$.

Table III presents the results of the bottom baryon masses. Similarly, the second-order $m_{s}$ corrections improve the results. The mass of the $\Omega_{b}^{*}$ is predicted to be $6081.9 \mathrm{MeV}$, whereas the model-independent approach of Ref. [22] predicts $M_{\Omega_{b}^{*}}=(6095 \pm 4.4) \mathrm{MeV}$. The difference is found to be less than $1 \%$.

TABLE II. Results of the masses of the charmed baryon masses in the unit of MeV. In the third and fourth columns, those with the first-order and second-order $m_{\mathrm{s}}$ corrections are listed. The last column represents the experimental data.

\begin{tabular}{llccc}
\hline \hline & & \multicolumn{2}{c}{$m_{\mathrm{s}}$ corrections } & \\
\cline { 3 - 4 } $\mathcal{R}_{J}^{Q}$ & $B_{c}$ & 1st order & 2nd order & Experiment \\
\hline$\overline{\mathbf{3}}_{1 / 2}^{c}$ & $\Lambda_{c}$ & 2274.4 & 2280.7 & $2286.5 \pm 0.1$ \\
$\overline{\mathbf{3}}_{1 / 2}^{c}$ & $\Xi_{c}$ & 2481.5 & 2475.2 & $2469.4 \pm 0.3$ \\
$\mathbf{6}_{1 / 2}^{c}$ & $\Sigma_{c}$ & 2455.7 & 2448.5 & $2453.5 \pm 0.1$ \\
$\mathbf{6}_{1 / 2}^{c}$ & $\Xi_{c}^{\prime}$ & 2575.2 & 2576.8 & $2576.8 \pm 2.1$ \\
$\mathbf{6}_{1 / 2}^{c}$ & $\Omega_{c}$ & 2694.6 & 2700.1 & $2695.2 \pm 1.7$ \\
$\mathbf{6}_{3 / 2}^{c}$ & $\Sigma_{c}^{*}$ & 2523.9 & 2516.7 & $2518.1 \pm 0.8$ \\
$\mathbf{6}_{3 / 2}^{c}$ & $\Xi_{c}^{*}$ & 2643.3 & 2645.0 & $2645.9 \pm 0.4$ \\
$\mathbf{6}_{3 / 2}^{c}$ & $\Omega_{c}^{*}$ & 2762.7 & 2768.3 & $2765.9 \pm 2.0$ \\
\hline \hline
\end{tabular}


TABLE III. Results of the masses of the bottom baryon masses in the unit of $\mathrm{MeV}$. In the third and fourth columns, those with the first-order and second-order $m_{\mathrm{s}}$ corrections are listed. The last column represents the experimental data.

\begin{tabular}{lcccc}
\hline \hline & & \multicolumn{2}{c}{$m_{\mathrm{s}}$ corrections } & \\
\cline { 3 - 4 } $\mathcal{R}_{J}^{Q}$ & $B_{b}$ & 1 st order & 2nd order & Experiment \\
\hline$\overline{\mathbf{3}}_{1 / 2}^{b}$ & $\Lambda_{b}$ & 5602.7 & 5609.0 & $5619.5 \pm 0.2$ \\
$\overline{\mathbf{3}}_{1 / 2}^{b}$ & $\Xi_{b}$ & 5809.9 & 5803.6 & $5793.1 \pm 0.7$ \\
$\boldsymbol{6}_{1 / 2}^{b}$ & $\Sigma_{b}$ & 5812.7 & 5805.5 & $5813.4 \pm 1.3$ \\
$\boldsymbol{6}_{1 / 2}^{b}$ & $\Xi_{b}^{\prime}$ & 5932.1 & 5933.8 & $5935.0 \pm 0.05$ \\
$\boldsymbol{6}_{1 / 2}^{b}$ & $\Omega_{b}$ & 6051.6 & 6057.1 & $6048.0 \pm 1.9$ \\
$\boldsymbol{6}_{3 / 2}^{b}$ & $\Sigma_{b}^{*}$ & 5834.7 & 5830.3 & $5833.6 \pm 1.3$ \\
$\boldsymbol{6}_{3 / 2}^{b}$ & $\Xi_{b}^{*}$ & 5954.2 & 5958.6 & $5955.3 \pm 0.1$ \\
$\mathbf{6}_{3 / 2}^{b}$ & $\Omega_{b}^{*}$ & 6073.6 & 6081.9 & $\ldots$ \\
\hline \hline
\end{tabular}

\section{SUMMARY AND CONCLUSIONS}

In the present work, we investigated the mass spectra of the lowest-lying singly heavy baryons within the framework of the self-consistent $\mathrm{SU}(3)$ chiral quark-soliton model. In the model, the $N_{c}-1$ light valence quarks polarize the Dirac sea. We obtained the soliton energy consisting of the $N_{c}-1$ valence-quark and sea-quark energies. Minimizing the soliton energy around the saddle point of the classical pion field self-consistently, we derived the soliton mass. Because of the hedgehog symmetry, we embedded the $\mathrm{SU}(2)$ soliton into the flavor $\mathrm{SU}(3)$. While we ignore the $1 / N_{c}$ quantum fluctuations in this mean-field approximation, the rotational zero modes or rotational $1 / N_{c}$ corrections are taken into account, a rigid rotation of the soliton being assumed. All the moments of inertia were computed in the present work explicitly.

We consider the effects of flavor SU(3) symmetry breaking to the second order in perturbation. As expected, the inclusion of the second-order $m_{\mathrm{s}}$ corrections leads to better results of the mass splittings of both the charmed and bottom heavy baryons than those with the linear $m_{\mathrm{s}}$ corrections, in comparison with the experimental data. Having fixed the center mass in each representation, we were able to obtain the numerical values of all the lowest-lying singly heavy baryons both in the charm and bottom sectors. With the second-order $m_{\mathrm{s}}$ corrections included, the present results are in very good agreement with the experimental data. The mass of the $\Omega_{b}^{*}$ baryon is predicted to be $6081.9 \mathrm{MeV}$ in the present work.

\section{ACKNOWLEDGMENTS}

We are grateful to M. V. Polyakov and M. Praszałowicz for valuable discussion. H.-C.K. wants to express his gratitude to A. Hosaka for information on the original paper by W. Pauli and S. M. Dancoff about the hedgehog ansatz. H.-C. K. is also thankful to the members of the Research Center for Nuclear Physics, Osaka University. The work of
H.-Ch. K. was supported by Basic Science Research Program through the National Research Foundation of Korea funded by the Ministry of Education, Science and Technology (Grant No. NRF-2015R1D1A1A01060707).

\section{APPENDIX A: MOMENTS OF INERTIA}

In this Appendix, we compile all relevant formulas for the modified $\pi N$ sigma term and the moments of inertia $I_{1,2}, K_{1,2}$, and $N_{1,2}$. All terms consist of the vacuum and sea parts. The modified $\pi N$ sigma term is written as

$$
\bar{\Sigma}_{\pi N}=\bar{\Sigma}_{\pi N}^{\mathrm{val}}+\Sigma_{\pi N}^{\mathrm{sea}},
$$

where the valence and sea parts are expressed, respectively, as

$$
\begin{aligned}
& \bar{\Sigma}_{\pi N}^{\mathrm{val}}=m_{0}\left(N_{c}-1\right)\left\langle\mathrm{val}\left|\gamma_{4}\right| \mathrm{val}\right\rangle, \\
& \Sigma_{\pi N}^{\mathrm{sea}}=\frac{m_{0}}{2} N_{c} \sum_{n}\left\langle n\left|\gamma_{4}\right| n\right\rangle \operatorname{sign}\left(E_{n}\right) \mathcal{R}_{\Sigma}\left(E_{n}\right),
\end{aligned}
$$

where $\gamma_{4}$ is the Dirac $\gamma$ matrix in Euclidean space represented as

$$
\gamma_{4}=\left(\begin{array}{cc}
\mathbf{1} & 0 \\
0 & -\mathbf{1}
\end{array}\right)
$$

The function $\mathcal{R}_{\Sigma}\left(E_{n}\right)$ denotes a regularization function written as

$$
\mathcal{R}_{\Sigma}\left(E_{n}\right)=\frac{1}{\sqrt{\pi}} \int_{0}^{\infty} \frac{d u}{\sqrt{u}} e^{-u} \phi\left(u / E_{n}^{2}\right),
$$

where $\phi(u)$ [34] is a cutoff function defined by

$$
\phi(u)=c \theta\left(u-1 / \Lambda_{1}^{2}\right)+(1-c) \theta\left(u-1 / \Lambda_{2}^{2}\right) .
$$

The free parameters $\Lambda_{1}, \Lambda_{2}$, and $c$ are determined in the mesonic sector by reproducing the pion decay constant $f_{\pi}=93 \mathrm{MeV}$ and the pion mass $m_{\pi}=139 \mathrm{MeV}$. Their numerical values are explicitly given as $\Lambda_{1}=381.15 \mathrm{MeV}$, $\Lambda_{2}=1428.00 \mathrm{MeV}$, and $c=0.7276$.

The moment of inertia tensor $I_{a b}$ is given as

$$
I_{\mathrm{ab}}=I_{\mathrm{ab}}^{\mathrm{val}}+I_{\mathrm{ab}}^{\mathrm{sea}},
$$

where

$$
\begin{aligned}
& I_{\mathrm{ab}}^{\mathrm{val}}=\frac{\left(N_{c}-1\right)}{2} \sum_{\text {val, } \mathrm{n} \neq \mathrm{val}} \frac{\left\langle n\left|\lambda_{a}\right| \mathrm{val}\right\rangle\left\langle\mathrm{val}\left|\lambda_{b}\right| n\right\rangle}{E_{n}-E_{\mathrm{val}}}, \\
& I_{\mathrm{ab}}^{\mathrm{sea}}=\frac{N_{c}}{4} \sum_{m, n}\left\langle n\left|\lambda_{a}\right| m\right\rangle\left\langle m\left|\lambda_{b}\right| n\right\rangle \mathcal{R}_{I}\left(E_{n}, E_{m}\right),
\end{aligned}
$$

with the different regularization function $R_{I}\left(E_{n}, E_{m}\right)$ 


$$
\begin{aligned}
\mathcal{R}_{I}\left(E_{n}, E_{m}\right)= & \frac{1}{2 \sqrt{\pi}} \int_{0}^{\infty} \frac{d u}{\sqrt{u}} \phi(u)\left[\frac{e^{-u E_{n}^{2}}-e^{-u E_{m}^{2}}}{u\left(E_{m}^{2}-E_{n}^{2}\right)}\right. \\
& \left.-\frac{E_{n} e^{-u E_{n}^{2}}+E_{m} e^{-u E_{m}^{2}}}{E_{m}+E_{n}}\right] .
\end{aligned}
$$

$\lambda_{a}$ in Eq. (A7) denote the Gell-Mann matrices for the flavor $\mathrm{SU}(3)$ group, satisfying $\operatorname{tr}\left(\lambda_{a} \lambda_{b}\right)=2 \delta_{a b}$ and $\left[\lambda_{a}, \lambda_{b}\right]=$ $2 i f_{a b c} \lambda_{c}, a=1, \ldots, 8$. The moments of inertia $I_{1}$ and $I_{2}$ are defined by

$$
I_{a b} \equiv\left\{\begin{array}{cl}
I_{1} \delta_{a b} & a, b=1,2,3 \\
I_{2} \delta_{a b} & a, b=4,5,6,7 \\
0 & a, b=8
\end{array}\right.
$$

Similarly, the anomalous moments-of-inertia tensor is expressed as

$$
K_{\mathrm{ab}}=K_{\mathrm{ab}}^{\mathrm{val}}+K_{\mathrm{ab}}^{\mathrm{sea}},
$$

where

$$
\begin{aligned}
K_{\mathrm{ab}}^{\mathrm{val}} & =\frac{\left(N_{c}-1\right)}{2} \sum_{\mathrm{val}, \mathrm{n} \neq \mathrm{val}} \frac{\left\langle n\left|\lambda_{a}\right| \mathrm{val}\right\rangle\left\langle\mathrm{val}\left|\lambda_{b} \gamma_{4}\right| n\right\rangle}{E_{n}-E_{\mathrm{val}}}, \\
K_{\mathrm{ab}}^{\mathrm{sea}} & =\frac{N_{c}}{8} \sum_{m, n}\left\langle n\left|\lambda_{a}\right| m\right\rangle\left\langle m\left|\gamma_{4} \lambda_{b}\right| n\right\rangle \frac{\operatorname{sign}\left(E_{n}\right)-\operatorname{sign}\left(E_{m}\right)}{E_{n}-E_{m}} .
\end{aligned}
$$

The anomalous moments of inertia $K_{1}$ and $K_{2}$ are defined by

$$
K_{a b} \equiv \begin{cases}K_{1} \delta_{a b} & a, b=1,2,3 \\ K_{2} \delta_{a b} & a, b=4,5,6,7 \\ 0 & a, b=8\end{cases}
$$

Finally, we express the third moments-of-inertia tensor, which appears only when the second-order $m_{\mathrm{s}}$ corrections are considered,

$$
N_{\mathrm{ab}}=N_{\mathrm{ab}}^{\mathrm{val}}+N_{\mathrm{ab}}^{\mathrm{sea}}
$$

Then the moment of inertia

$N_{\mathrm{ab}}^{\mathrm{val}}=\frac{\left(N_{c}-1\right)}{2} \sum_{\text {val, } \mathrm{n} \neq \mathrm{val}} \frac{\left\langle n\left|\lambda_{a} \gamma_{4}\right| \mathrm{val}\right\rangle\left\langle\mathrm{val}\left|\lambda_{b} \gamma_{4}\right| n\right\rangle}{E_{n}-E_{\mathrm{val}}}$,

$N_{\mathrm{ab}}^{\mathrm{sea}}=\frac{N_{c}}{4} \sum_{m, n}\left\langle n\left|\lambda_{a} \gamma_{4}\right| m\right\rangle\left\langle m\left|\lambda_{b} \gamma_{4}\right| n\right\rangle \mathcal{R}_{N}\left(E_{n}, E_{m}\right)$,

with the regularization function

$$
\mathcal{R}_{N}\left(E_{n}, E_{m}\right)=\frac{1}{2 \sqrt{\pi}} \int_{0}^{\infty} \frac{d u}{\sqrt{u}} \phi(u) \frac{E_{n} e^{-u E_{n}^{2}}-E_{m} e^{-u E_{m}^{2}}}{E_{n}-E_{m}} .
$$

$N_{0}, N_{1}$, and $N_{2}$ are defined by

$$
N_{a b} \equiv \begin{cases}N_{1} \delta_{a b} & a, b=1,2,3 \\ N_{2} \delta_{a b} & a, b=4,5,6,7 . \\ \frac{1}{3} N_{0} & a, b=8\end{cases}
$$

\section{APPENDIX B: MATRIX ELEMENTS OF THE SU(3) WIGNER $D$ FUNCTIONS}

In this Appendix, we tabulate in Tables IV-VI all relevant matrix elements of the SU(3) Wigner $D$ functions in each representation.

TABLE IV. Matrix elements of the SU(3) Wigner $D$ functions $D_{88}^{(8)}$ and $D_{8 i}^{(8)} J_{i}$.

\begin{tabular}{cccccc}
\hline \hline & $\mathcal{R}$ & $\mathrm{T}$ & $\mathrm{Y}$ & $\left\langle\mathcal{R} Y T J\left|D_{88}^{(8)}\right| \mathcal{R} Y T J\right\rangle$ & $\left\langle\mathcal{R} Y T J\left|D_{8 i}^{(8)} J_{i}\right| \mathcal{R} Y T J\right\rangle$ \\
\hline$\Lambda_{c}$ & $\overline{\mathbf{3}}$ & 0 & $2 / 3$ & $1 / 4$ & 0 \\
$\Xi_{c}$ & & $1 / 2$ & $-1 / 3$ & $-1 / 8$ & 0 \\
$\Sigma_{c}$ & $\mathbf{6}$ & 1 & $2 / 3$ & $1 / 10$ & $-\sqrt{3} / 5$ \\
$\Xi_{c}$ & & $1 / 2$ & $-1 / 3$ & $-1 / 20$ & $\sqrt{3} / 10$ \\
$\Omega_{c}$ & & 0 & $-4 / 3$ & $-1 / 5$ & $2 \sqrt{3} / 5$ \\
\hline \hline
\end{tabular}


TABLE V. Matrix elements of the products of the SU(3) Wigner $D$ functions, $D_{88}^{(8)} D_{88}^{(8)}, D_{8 i}^{(8)} D_{8 i}^{(8)}$, and $D_{8 p}^{(8)} D_{8 p}^{(8)}$, where index $i$ runs from 1 to 3 , whereas $p$ runs from 4 to 7 .

\begin{tabular}{ccccccc}
\hline \hline & $\mathcal{R}$ & $\mathrm{T}$ & $\mathrm{Y}$ & $\left\langle\mathcal{R} Y T J\left|D_{88}^{(8)} D_{88}^{(8)}\right| \mathcal{R} Y T J\right\rangle$ & $\left\langle\mathcal{R} Y T J\left|D_{8 i}^{(8)} D_{8 i}^{(8)}\right| \mathcal{R} Y T J\right\rangle$ & $\left\langle\mathcal{R} Y T J\left|D_{8 p}^{(8)} D_{8 p}^{(8)}\right| \mathcal{R} Y T J\right\rangle$ \\
\hline$\Lambda_{c}$ & $\overline{\mathbf{3}}$ & 0 & $2 / 3$ & $7 / 40$ & $9 / 40$ & $3 / 5$ \\
$\Xi_{c}$ & & $1 / 2$ & $-1 / 3$ & $1 / 10$ & $9 / 20$ & $9 / 20$ \\
$\Sigma_{c}$ & $\mathbf{6}$ & 1 & $2 / 3$ & $3 / 20$ & $19 / 60$ & $8 / 15$ \\
$\Xi_{c}$ & & $1 / 2$ & $-1 / 3$ & $1 / 10$ & $2 / 5$ & $1 / 2$ \\
$\Omega_{c}$ & 0 & $-4 / 3$ & $1 / 10$ & $1 / 2$ & $2 / 5$ \\
\hline \hline
\end{tabular}

TABLE VI. Transition matrix elements of the SU(3) Wigner $D$ functions $D_{88}^{(8)}$ and $D_{8 i}^{(8)} J_{i}$, which appear from the second-order perturbation.

\begin{tabular}{lcccccc}
\hline \hline & $\mathcal{R}^{\prime}$ & $\mathcal{R}$ & $\mathrm{T}$ & $\mathrm{Y}$ & $\left\langle\mathcal{R}^{\prime} Y T J\left|D_{88}^{(8)}\right| \mathcal{R} Y T J\right\rangle$ & $\left\langle\mathcal{R}^{\prime} Y T J\left|D_{8 i}^{(8)} J_{i}\right| \mathcal{R} Y T J\right\rangle$ \\
\hline$\Lambda_{c}$ & $\mathbf{1 5}$ & $\overline{\mathbf{3}}$ & 0 & $2 / 3$ & $3 \sqrt{5} / 20$ & 0 \\
$\Xi_{c}$ & & & $1 / 2$ & $-1 / 3$ & $3 \sqrt{15} / 40$ & 0 \\
$\Sigma_{c}$ & $\overline{\mathbf{1 5}}$ & $\mathbf{6}$ & 1 & $2 / 3$ & $\sqrt{10} / 10$ & $\sqrt{30} / 15$ \\
$\Xi_{c}$ & & & $1 / 2$ & $-1 / 3$ & $\sqrt{15} / 20$ & $\sqrt{5} / 10$ \\
$\Omega_{c}$ & & & 0 & $-4 / 3$ & 0 & 0 \\
$\Sigma_{c}$ & $\overline{\mathbf{2 4}}$ & $\mathbf{6}$ & 1 & $2 / 3$ & $1 / 5$ & $-\sqrt{3} / 15$ \\
$\Xi_{c}$ & & & $1 / 2$ & $-1 / 3$ & $\sqrt{6} / 10$ & $-\sqrt{2} / 10$ \\
$\Omega_{c}$ & & & 0 & $-4 / 3$ & $\sqrt{6} / 10$ & $-\sqrt{2} / 10$ \\
\hline \hline
\end{tabular}

[1] T. Aaltonen et al. (CDF Collaboration), Phys. Rev. Lett. 99, 202001 (2007).

[2] S. Chatrchyan et al. (CMS Collaboration), Phys. Rev. Lett. 108, 252002 (2012).

[3] V. M. Abazov et al. (D0 Collaboration), Phys. Rev. Lett. 101, 232002 (2008).

[4] T. Kuhr (CDF and D0 Collaborations), arXiv:1109.1944.

[5] R. Aaij et al. (LHCb Collaboration), Phys. Rev. Lett. 109, 172003 (2012).

[6] R. Aaij et al. (LHCb Collaboration), Phys. Rev. Lett. 110, 182001 (2013).

[7] R. Aaij et al. (LHCb Collaboration), Phys. Rev. Lett. 113, 032001 (2014).

[8] R. Aaij et al. (LHCb Collaboration), Phys. Rev. Lett. 113, 242002 (2014).

[9] R. Aaij et al. (LHCb Collaboration), Phys. Rev. Lett. 114, 062004 (2015).

[10] R. Aaij et al. (LHCb Collaboration), Phys. Rev. Lett. 118, 182001 (2017).

[11] N. Isgur and M. B. Wise, Phys. Lett. B 232, 113 (1989).

[12] N. Isgur and M. B. Wise, Phys. Rev. Lett. 66, 1130 (1991).

[13] H. Georgi, Phys. Lett. B 240, 447 (1990).

[14] C. G. Callan, Jr. and I. R. Klebanov, Nucl. Phys. B262, 365 (1985).

[15] C. G. Callan, Jr., K. Hornbostel, and I. R. Klebanov, Phys. Lett. B 202, 269 (1988).

[16] M. Rho, D. O. Riska, and N. N. Scoccola, Phys. Lett. B 251, 597 (1990).
[17] E. E. Jenkins, A. V. Manohar, and M. B. Wise, Nucl. Phys. B396, 27 (1993).

[18] Z. Guralnik, M. E. Luke, and A. V. Manohar, Nucl. Phys. B390, 474 (1993).

[19] K. S. Gupta, M. A. Momen, J. Schechter, and A. Subbaraman, Phys. Rev. D 47, R4835 (1993).

[20] A. Momen, J. Schechter, and A. Subbaraman, Phys. Rev. D 49, 5970 (1994).

[21] Y. s. Oh, B. Y. Park, and D. P. Min, Phys. Rev. D 50, 3350 (1994).

[22] G.-S. Yang, H.-C. Kim, M. V. Polyakov, and M. Praszałowicz, Phys. Rev. D 94, 071502 (2016).

[23] D. Diakonov, arXiv:1003.2157.

[24] E. Witten, Nucl. Phys. B160, 57 (1979).

[25] E. Witten, Nucl. Phys. B223, 422 (1983); B223, 433 (1983).

[26] W. Pauli and S. M. Dancoff, Phys. Rev. 62, 85 (1942).

[27] T. H. R. Skyrme, Proc. R. Soc. A 260, 127 (1961).

[28] E. Guadagnini, Nucl. Phys. B236, 35 (1984).

[29] P. O. Mazur, M. A. Nowak, and M. Praszałowicz, Phys. Lett. 147B, 137 (1984).

[30] S. Jain and S. R. Wadia, Nucl. Phys. B258, 713 (1985).

[31] D. Diakonov, V. Y. Petrov, and P. V. Pobylitsa, Nucl. Phys. B306, 809 (1988).

[32] C. V. Christov, A. Blotz, H.-C. Kim, P. Pobylitsa, T. Watabe, T. Meissner, E. R. Arriola, and K. Goeke, Prog. Part. Nucl. Phys. 37, 91 (1996).

[33] D. Diakonov, arXiv:hep-ph/9802298. 
[34] A. Blotz, D. Diakonov, K. Goeke, N. W. Park, V. Petrov, and P. V. Pobylitsa, Nucl. Phys. A555, 765 (1993).

[35] H.-C. Kim, A. Blotz, M. V. Polyakov, and K. Goeke, Phys. Rev. D 53, 4013 (1996).

[36] A. Silva, H.-C. Kim, and K. Goeke, Phys. Rev. D 65, 014016 (2001); 66, 039902(E) (2002).

[37] T. Ledwig, A. Silva, H.-C. Kim, and K. Goeke, J. High Energy Phys. 07 (2008) 132.

[38] T. Ledwig, A. Silva, and H.-C. Kim, Phys. Rev. D 82, 034022 (2010).
[39] D. Diakonov, V. Petrov, P. Pobylitsa, M. V. Polyakov, and C. Weiss, Nucl. Phys. B480, 341 (1996).

[40] H.-C. Kim, M. V. Polyakov, and M. Praszalowicz, Phys. Rev. D 96, 014009 (2017).

[41] A. Blotz, Strangeness in the semibosonized Nambu-JonaLasinio model, Ph.D. thesis, Ruhr-University Bochum, 1993 (unpublished).

[42] H.-C. Kim, M. V. Polyakov, M. Praszałowicz, and G. S. Yang, Phys. Rev. D 96, 094021 (2017); 97, 039901(E) (2018).

[43] G. S. Yang and H.-C. Kim, Phys. Lett. B 781, 601 (2018). 